\title{
Some Computations Regarding Foulkes' Conjecture
}

\author{
Jurgen Müller and Max Neunhöffer
}

\section{CONTENTS}

1. Foulkes' Conjecture

2. Implementation of the action of $S_{m n}$ on $\Omega_{m, n}$

3. Schur Bases

4. The Approach of Black and List

5. The Computational Approach

6. Actual Computations

Addendum

References
2000 AMS Subject Classification: Primary 05E10;

Secondary 20C30, 20C40

Keywords: Foulkes' conjecture, symmetric group, representations of symmetric groups, plythysm, wreath product
We describe how certain permutation actions of large symmetric groups can be efficiently implemented on a computer. Using a specially tailored adaptation of a general technique to enumerate huge orbits and substantial distributed computation on a cluster of workstations, we collect further evidence related to the approach to Foulkes' conjecture suggested in [Black and List 89].

\section{FOULKES' CONJECTURE}

To state Foulkes' conjecture we first introduce some notation. Let $\mathbb{N}$ be the set of positive integers, let $\mathbb{Q}$ be the set of rational numbers, and denote by $M_{n}:=\{1,2,3, \ldots, n\}$ for $n \in \mathbb{N}$ the set of natural numbers less than or equal to $n$. We denote the symmetric group on $n$ points by $S_{n}:=\left\{\pi: M_{n} \rightarrow M_{n} \mid \pi\right.$ bijective $\}$, with concatenation of maps as product, which we denote as $\pi \circ \varphi$, meaning "first apply $\varphi$, then $\pi$."

For $m, n \in \mathbb{N}$ let $S_{m} \imath S_{n}$ be the wreath product of $S_{m}$ and $S_{n}$, which is a semidirect product of the $n$-fold direct product $S_{m}^{n}:=S_{m} \times \cdots \times S_{m}$ of copies of $S_{m}$ and $S_{n}$, where the latter acts on the first by permuting the direct factors. Note that $S_{m}^{n}$ can be identified with the set of $\operatorname{maps}\left\{f: M_{n} \rightarrow S_{m}\right\}$. Hence, $S_{m} \prec S_{n}=S_{m}^{n} \rtimes S_{n}$ with product

$$
(f, \pi) \cdot\left(f^{\prime}, \pi^{\prime}\right):=\left(f \cdot\left(f^{\prime} \circ \pi^{-1}\right), \pi \circ \pi^{\prime}\right),
$$

where we multiply maps $f: M_{n} \rightarrow S_{m}$ pointwise using the product in $S_{m}$.

The wreath product $S_{m}<S_{n}$ has order $\left|S_{m}<S_{n}\right|=$ $(m !)^{n} \cdot n$ ! and embeds into $S_{m n}$ by letting the $i$ th direct factor of $S_{m}^{n}$, for $i=1, \ldots, n$, permute the points $\{(i-1) m+1, \ldots, i m\}$ and keep all other points in $M_{m n}$ fixed, while $S_{n}$ acts on $M_{m n}$ by permuting these $n$ blocks; for more details see [James and Kerber 81, Section 4.1]. We denote by $\Omega_{m, n}$ the set $\left\{\left(S_{m} \prec S_{n}\right) \circ \pi \mid \pi \in S_{m n}\right\}$ of right cosets of $S_{m} \prec S_{n}$ in $S_{m n}$ and by $\mathbb{Q} \Omega_{m, n}$ the associated permutation right $\mathbb{Q} S_{m n}$-module.

(c) A K Peters, Ltd. $1058-6458 / 2005 \$ 0.50$ per page Experimental Mathematics 14:3, page 277 
It is easily seen by an induction argument that for $m \geq n$ we have $\left|S_{n} \prec S_{m}\right| \leq\left|S_{m} \prec S_{n}\right|$. Thus we have $\left|\Omega_{m, n}\right| \leq\left|\Omega_{n, m}\right|$. But in fact much more is conjectured to be true:

Conjecture 1.1. [Foulkes 50] Let $m, n \in \mathbb{N}$ with $m \geq n$. Then the permutation module $\mathbb{Q} \Omega_{m, n}$ is a $\mathbb{Q} S_{m n}$-submodule of the permutation module $\mathbb{Q} \Omega_{n, m}$.

In Section 2, we describe how the action of $S_{m n}$ on $\Omega_{m, n}$ can be efficiently implemented on a computer. This implementation will be used for calculations connected to the approach to Foulkes' conjecture suggested in [Black and List 89]. Our description uses the notion of Schur bases, which are introduced in Section 3, while in Section 4 , the approach of Black and List is discussed. In Section 5 , our particular computational techniques are explained and in Section 6, actual computational results are presented. There we also describe the values of $m$ and $n$ for which the conjecture has been verified computationally so far.

\section{IMPLEMENTATION OF THE ACTION OF $S_{m n}$ ON $\Omega_{m, n}$}

For this section, let $m, n \in \mathbb{N}$ be fixed. We consider the following set of maps:

$$
\begin{array}{r}
V_{m, n}:=\left\{v: M_{m n} \rightarrow M_{n} \mid v\right. \text { takes every value } \\
\text { exactly } m \text { times }\} .
\end{array}
$$

One can imagine these maps as tuples of length $m n$ with entries in $M_{n}$, each one occuring exactly $m$ times. Hence we will denote such maps as tuples $v=\left(v_{1}, v_{2}, \ldots, v_{m n}\right)$. On the computer they are stored exactly in this way. By way of concatenation of maps, we have two transitive actions on $V_{m, n}$, one on the left and one on the right. The group $S_{n}$ acts regularly on the left by renaming the entries:

$$
S_{n} \times V_{m, n} \rightarrow V_{m, n},(\pi, v) \mapsto \pi \circ v .
$$

The group $S_{m n}$ acts on the right by permuting the entries:

$$
V_{m, n} \times S_{m n} \rightarrow V_{m, n},(v, \psi) \mapsto v \circ \psi
$$

These actions commute because of the associativity of concatenation: $(\pi \circ v) \circ \psi=\pi \circ(v \circ \psi)$.

Therefore we obtain an induced action of $S_{m n}$ on the $S_{n}$-orbits in $V_{m, n}$. In the following, we omit the "o" symbol in the notation of $S_{n}$ orbits, denote the set $\left\{S_{n} v \mid\right.$ $\left.v \in V_{m, n}\right\}$ of $S_{n}$-orbits in $V_{m, n}$ by $S_{n} \backslash V_{m, n}$, and the action of $S_{m n}$ on it by $\left(S_{n} v\right) \circ \psi:=S_{n}(v \circ \psi)$.

From now on let $x \in V_{m, n}$ be the tuple

$$
x:=(\underbrace{1, \ldots, 1}_{m \text { times }}, \underbrace{2, \ldots, 2}_{m \text { times }}, \ldots, \underbrace{n, \ldots, n}_{m \text { times }}),
$$

i.e., the map that maps $k \in M_{m n}$ to $\lceil k / m\rceil$, the smallest integer greater or equal to $k / m$. Then the stabilizer $\operatorname{Stab}_{S_{m n}}(x)$ of $x$ in $S_{m n}$ is equal to $S_{m}^{n}$, and the stabilizer $\operatorname{Stab}_{S_{m n}}\left(S_{n} x\right)$ of $S_{n} x \in S_{n} \backslash V_{m, n}$ in $S_{m n}$ is equal to $S_{m} \prec S_{n}$. Thus the action of $S_{m n}$ on $S_{n} \backslash V_{m, n}$ is equivalent to the action of $S_{m n}$ on $\Omega_{m, n}$. Hence we identify $\Omega_{m, n}$ and $S_{n} \backslash V_{m, n}$ from now on.

Passing from $S_{m n}$-sets to $\mathbb{Q} S_{m n}$-modules, we can consider $\mathbb{Q} V_{m, n}$ as a $\mathbb{Q} S_{n}-\mathbb{Q} S_{m n}$-bimodule, and thus the permutation $\mathbb{Q} S_{m n}$-module $\mathbb{Q} \Omega_{m, n}$ is identified with the $\mathbb{Q} S_{m n}$-submodule $\left(\mathbb{Q} V_{m, n}\right)^{S_{n}}$ whose permutation basis consists of the sums $\overline{S_{n} v}:=\sum_{w \in S_{n} v} w$ over $S_{n}$-orbits $S_{n} v \subseteq V_{m, n}$. Note that $\left(\mathbb{Q} V_{m, n}\right)^{S_{n}}$ is the set of elements in $\mathbb{Q} V_{m, n}$ invariant under the left action of $S_{n}$.

We introduce the following definition to distinguish one tuple in each $S_{n}$-orbit.

Definition 2.1. ( $S_{n}$-Minimal Tuples.) In the above situation, we call the lexicographically smallest tuple in each $S_{n}$-orbit $S_{n}$-minimal. For each $v \in V_{m, n}$, we call the $S_{n^{-}}$ minimal tuple in the orbit $S_{n} v$ the $S_{n}$-minimalization of $v$. We denote by $V_{m, n}^{\min }$ the set of $S_{n}$-minimal tuples in $V_{m, n}$.

It follows readily from the above, that the action of $S_{m n}$ on $\Omega_{m, n}$ can be implemented on a computer by identifying $\Omega_{m, n}$ with $V_{m, n}^{\min }$ and acting with a map $\psi \in S_{m n}$ on $v \in V_{m, n}^{\min }$ by just $S_{n}$-minimalizing $v \circ \psi \in V_{m, n}$. Note the runtime needed to compute an $S_{n}$-minimalization, and hence the $\psi$-image of $v$, is proportional to the length $m n$ of the tuples.

We note the following characterization of $S_{n^{-}}$ minimality for later reference.

Proposition 2.2. (Equivalent Characterization of $\boldsymbol{S}_{\boldsymbol{n}}$ Minimality.) A tuple $v \in V_{m, n}$ is $S_{n}$-minimal, if and only if it has the following property: for all $i, j$ with $1 \leq i<j \leq n$, the first occurrence of $i$ in $v$ is before the first occurrence of $j$.

Proof: Let $v$ be $S_{n}$-minimal. If the above property does not hold, we can rename some $i$ and $j$ and get a lexicographically smaller tuple in the same $S_{n}$-orbit; a contradiction. 
Let $v$ have the above property, and assume $v$ is not $S_{n}$-minimal. Then there is a tuple $v^{\prime}$ in the same $S_{n^{-}}$ orbit that is lexicographically smaller than $v$. Let $p$ be the first position where both tuples differ, and let $v_{p}=j$ and $v_{p}^{\prime}=i$ with $i<j$. Because $v$ and $v^{\prime}$ are in the same $S_{n}$-orbit, $p$ is the first position in $v$ with value $j$ and the first position in $v^{\prime}$ with value $i$. By the assumed property, the first occurrence of $i$ in $v$ is before $p$. However, $v$ and $v^{\prime}$ are equal at positions before $p$; therefore we have a contradiction.

\section{SCHUR BASES}

To describe the approach in [Black and List 89], we recall a few facts about permutation modules and homomorphisms between them. For our purposes, we give a slightly more general description than can be found in [Landrock 83, Chapter II.12].

For this section, let $G$ be a finite group, acting transitively from the right on the sets $\Omega$ and $\Omega^{\prime}$. Let $\omega_{1} \in \Omega$ and $\omega_{1}^{\prime} \in \Omega^{\prime}$, and let $H:=\operatorname{Stab}_{G}\left(\omega_{1}\right)$ and $H^{\prime}:=\operatorname{Stab}_{G}\left(\omega_{1}^{\prime}\right)$ be the corresponding stabilizers. As above, let $\mathbb{Q} \Omega$ and $\mathbb{Q} \Omega^{\prime}$ denote the associated permutation modules. The space $\operatorname{Hom}_{\mathbb{Q} G}\left(\mathbb{Q} \Omega, \mathbb{Q} \Omega^{\prime}\right)$ of $\mathbb{Q} G$ homomorphisms from $\mathbb{Q} \Omega$ to $\mathbb{Q} \Omega^{\prime}$ has a distinguished basis, which can be described as follows:

We decompose $\Omega^{\prime}$ into $H$-orbits, by choosing $s_{1}=$ $1_{G}, s_{2}, \ldots, s_{l} \in G$ such that

$$
\Omega^{\prime}=\omega_{1}^{\prime} s_{1} H \cup \omega_{1}^{\prime} s_{2} H \cup \cdots \cup \omega_{1}^{\prime} s_{l} H
$$

is a disjoint union. Note that $\left\{s_{1}, s_{2}, \ldots, s_{l}\right\}$ is a set of $H^{\prime}$-H-double coset representatives in $G$.

Using the diagonal action of $G$ on $\Omega^{\prime} \times \Omega$ and considering the intersection of each $G$-orbit in $\Omega^{\prime} \times \Omega$ with $\Omega^{\prime} \times\left\{\omega_{1}\right\}$, we get the decomposition of $\Omega^{\prime} \times \Omega$ into $G$ orbits:

$$
\Omega^{\prime} \times \Omega=\left(\omega_{1}^{\prime} s_{1}, \omega_{1}\right) G \cup\left(\omega_{1}^{\prime} s_{2}, \omega_{1}\right) G \cup \cdots \cup\left(\omega_{1}^{\prime} s_{l}, \omega_{1}\right) G .
$$

We describe a homomorphism $\varphi \in \operatorname{Hom}_{\mathbb{Q} G}\left(\mathbb{Q} \Omega, \mathbb{Q} \Omega^{\prime}\right)$ by a matrix with respect to the natural bases of $\mathbb{Q} \Omega$, respectively $\mathbb{Q} \Omega^{\prime}$, where the rows are indexed by $\Omega^{\prime}$ and the columns are indexed by $\Omega$. Denoting the $\left(\omega^{\prime}, \omega\right)$-entry of the matrix of $\varphi$ by $\varphi_{\omega^{\prime}, \omega}$, we get $\varphi_{\omega^{\prime}, \omega g}=\varphi_{\omega^{\prime} g^{-1}, \omega}$, or equivalently $\varphi_{\omega^{\prime} g, \omega g}=\varphi_{\omega^{\prime}, \omega}$, for all $\omega \in \Omega, \omega^{\prime} \in \Omega^{\prime}$, and $g \in G$, because $\varphi$ is a $\mathbb{Q} G$-module homomorphism. Thus, the matrix of $\varphi$ is a unique $\mathbb{Q}$-linear combination of the matrices $A^{(1)}, A^{(2)}, \ldots, A^{(l)}$ defined by

$$
A_{\omega^{\prime}, \omega}^{(i)}= \begin{cases}1 & \text { if }\left(\omega^{\prime}, \omega\right) \in\left(\omega_{1}^{\prime} s_{i}, \omega_{1}\right) G, \\ 0 & \text { if }\left(\omega^{\prime}, \omega\right) \notin\left(\omega_{1}^{\prime} s_{i}, \omega_{1}\right) G .\end{cases}
$$

We call $\mathcal{A}:=\left(A^{(1)}, A^{(2)}, \ldots, A^{(l)}\right)$ and the associated $\mathbb{Q} G$-module homomorphisms $\left(\varphi^{(1)}, \varphi^{(2)}, \ldots, \varphi^{(l)}\right)$ the Schur basis of $\operatorname{Hom}_{\mathbb{Q} G}\left(\mathbb{Q} \Omega, \mathbb{Q} \Omega^{\prime}\right)$, which hence is in bijection with the $G$-orbits in $\Omega^{\prime} \times \Omega$. In particular, for $\omega=\omega_{1} g \in \Omega$, where $g \in G$, and thus $H^{g}=\operatorname{Stab}_{G}\left(\omega_{1} g\right)$, we have

$$
\varphi^{(i)}: \omega=\omega_{1} g \mapsto \sum_{\omega^{\prime} \in \omega_{1}^{\prime} s_{i} g H^{g}} \omega^{\prime} .
$$

We now turn to the concatenation of homomorphisms. For a $G$-set $\Omega^{\prime \prime}$, let $H^{\prime \prime}:=\operatorname{Stab}_{G}\left(\omega_{1}^{\prime \prime}\right)$ for some $\omega_{1}^{\prime \prime} \in \Omega^{\prime \prime}$, and as above we choose a set $\left\{t_{1}=1_{G}, t_{2}, \ldots\right\}$ of $H^{\prime \prime}-H^{\prime}$-double coset representatives in $G$ and a set $\left\{u_{1}=1_{G}, u_{2}, \ldots\right\}$ of $H^{\prime \prime}$ - $H$-double coset representatives in $G$. Let $\mathcal{B}:=\left(B^{(1)}, B^{(2)}, \ldots\right)$ and $\mathcal{C}:=\left(C^{(1)}, C^{(2)}, \ldots\right)$ denote the Schur bases of $\operatorname{Hom}_{\mathbb{Q} G}\left(\mathbb{Q} \Omega^{\prime}, \mathbb{Q} \Omega^{\prime \prime}\right)$ and $\operatorname{Hom}_{\mathbb{Q} G}\left(\mathbb{Q} \Omega, \mathbb{Q} \Omega^{\prime \prime}\right)$, respectively. We can now write the concatenation $B^{(j)} \circ A^{(i)}$, i.e., the matrix product, in terms of the Schur basis $\mathcal{C}$ of $\operatorname{Hom}_{\mathbb{Q} G}\left(\mathbb{Q} \Omega, \mathbb{Q} \Omega^{\prime \prime}\right)$ :

$$
\begin{aligned}
\left(B^{(j)} \circ A^{(i)}\right)_{\omega_{1}^{\prime \prime} u_{k}, \omega_{1}}=\sum_{\omega^{\prime} \in \Omega^{\prime}} B_{\omega_{1}^{\prime \prime} u_{k}, \omega^{\prime}}^{(j)} \cdot A_{\omega^{\prime}, \omega_{1}}^{(i)} \\
=\mid\left\{\omega^{\prime} \in \Omega^{\prime} \mid\left(\omega_{1}^{\prime \prime} u_{k}, \omega^{\prime}\right) \in\left(\omega_{1}^{\prime \prime} t_{j}, \omega_{1}^{\prime}\right) G\right. \\
\left.\quad \text { and }\left(\omega^{\prime}, \omega_{1}\right) \in\left(\omega_{1}^{\prime} s_{i}, \omega_{1}\right) G\right\} \mid \\
=\left|\left\{\omega^{\prime} \in \omega_{1}^{\prime} s_{i} H \mid\left(\omega_{1}^{\prime \prime} u_{k}, \omega^{\prime}\right) \in\left(\omega_{1}^{\prime \prime} t_{j}, \omega_{1}^{\prime}\right) G\right\}\right| \\
=\left|\left\{\omega^{\prime} \in \omega_{1}^{\prime} s_{i} H \mid\left(\omega_{1}^{\prime \prime}, \omega^{\prime} u_{k}^{-1}\right) \in\left(\omega_{1}^{\prime \prime}, \omega_{1}^{\prime} t_{j}^{-1}\right) G\right\}\right| \\
=\left|\omega_{1}^{\prime} s_{i} H u_{k}^{-1} \cap \omega_{1}^{\prime} t_{j}^{-1} H^{\prime \prime}\right| \\
=\left|\omega_{1}^{\prime} s_{i} H \cap \omega_{1}^{\prime} t_{j}^{-1} H^{\prime \prime} u_{k}\right| .
\end{aligned}
$$

\section{THE APPROACH OF BLACK AND LIST}

In [Black and List 89], the authors describe an approach to prove Foulkes' conjecture that is based on a certain $\mathbb{Q} S_{m n}$-module homomorphism $\varphi^{(m, n)}: \mathbb{Q} \Omega_{m, n} \rightarrow$ $\mathbb{Q} \Omega_{n, m}$. Using the language of the previous section, we first introduce a $\mathbb{Q} S_{m n}$-module homomorphism $\widetilde{\varphi}^{(m, n)}$ : $\mathbb{Q} V_{m, n} \rightarrow \mathbb{Q} V_{n, m}$, with a view towards efficient implementation.

For a tuple $v \in V_{m, n}$ let $\tilde{v}:=\left(\tilde{v}_{1}, \tilde{v}_{2}, \ldots, \tilde{v}_{m n}\right)$, where $\tilde{v}_{k}:=\left|\left\{l \in M_{k} \mid v_{l}=v_{k}\right\}\right|$. With respect to $v$, the tuple $\tilde{v}$ has the following property:

for every $i \in M_{n}$ and every $j \in M_{m}$, there is a position $K \in M_{m n}$ with $v_{k}=i$ and $\tilde{v}_{k}=j$.

From this property, it follows that $\tilde{v} \in V_{n, m}$. Obviously, the set of all such tuples coincides with $\tilde{v} \circ \operatorname{Stab}_{S_{m n}}(v) \subseteq$ $V_{n, m}$, and $\tilde{v}$ is the lexicographically smallest of them. In 
particular, we have

$$
\tilde{x}=(\underbrace{\underbrace{1,2, \ldots, m}, \underbrace{1,2, \ldots, m}, \ldots, \underbrace{1,2, \ldots, m}}_{n \text { times }} .
$$

For $v \in V_{m, n}$ and $i=1, \ldots, n$ let $1 \leq p_{i, 1}<p_{i, 2}<$ $\cdots<p_{i, m} \leq m n$ be the positions such that $v_{p_{i, j}}=i$, and let $\psi_{v} \in S_{m n}$ be defined as $\psi_{v}: p_{i, j} \mapsto(i-1) m+j$, for $i=$ $1, \ldots, n$ and $j=1, \ldots, m$. Hence we have $x \circ \psi_{v}=v$ and $\tilde{x} \circ \psi_{v}=\tilde{v}$. Thus we conclude that all pairs $(\tilde{v}, v)$, for $v \in$ $V_{m, n}$, belong to one and the same $G$-orbit in $V_{n, m} \times V_{m, n}$, and hence let $\widetilde{\varphi}^{(m, n)} \in \operatorname{Hom}_{\mathbb{Q} S_{m n}}\left(\mathbb{Q} V_{m, n}, \mathbb{Q} V_{n, m}\right)$ be the corresponding Schur basis element. As $\operatorname{Stab}_{S_{m n}}(x)=S_{m}^{n}$ acts regularly on its orbit $\tilde{x} \circ S_{m}^{n} \subseteq V_{n, m}$, for $v \in V_{m, n}$, we have

$$
\widetilde{\varphi}^{(m, n)}: v \mapsto \sum_{w \in \tilde{v} \circ \operatorname{Stab}_{S_{m n}}(v)} w=\sum_{\eta \in \operatorname{Stab}_{S_{m n}}(v)} \tilde{v} \circ \eta .
$$

Note that, if $\sigma_{m, n} \in S_{m n}$ is defined as

$$
\sigma_{m, n}:(i-1) m+j \mapsto(j-1) n+i
$$

for $i=1, \ldots, n$ and $j=1, \ldots, m$, then $\widetilde{\varphi}^{(m, n)}$ is the Schur basis element corresponding to the $S_{m}^{n}-S_{n}^{m}$-double coset $S_{m}^{n} \circ \sigma_{m, n} \circ S_{n}^{m}$ in $S_{m n}$.

Next we consider $\mathbb{Q} \Omega_{m, n}=\left(\mathbb{Q} V_{m, n}\right)^{S_{n}}$ and $\mathbb{Q} V_{n, m}=$ $\left(\mathbb{Q} V_{n, m}\right)^{S_{m}}$. By the description $(\star)$ of the elements of $\tilde{v} \circ \operatorname{Stab}_{S_{m n}}(v) \subseteq V_{n, m}$, for $v \in V_{m, n}$, we conclude that $\tilde{v} \circ$ $\operatorname{Stab}_{S_{m n}}(v)$ is a union of $S_{m}$-orbits. Hence by restriction, we obtain a $\mathbb{Q} S_{m n}$-homomorphism

$$
\varphi^{(m, n)}:=\left.\frac{1}{n !} \cdot \widetilde{\varphi}^{(m, n)}\right|_{\mathbb{Q} \Omega_{m, n}}: \mathbb{Q} \Omega_{m, n} \rightarrow \mathbb{Q} \Omega_{n, m} .
$$

Moreover, as for $v^{\prime}:=\pi \circ v$, for $\pi \in S_{n}$, we have $\widetilde{v^{\prime}}=\tilde{v}$ and $\operatorname{Stab}_{S_{m n}}(v)=\operatorname{Stab}_{S_{m n}}\left(v^{\prime}\right)$; we conclude that $\widetilde{\varphi}^{(m, n)}\left(v^{\prime}\right)=\widetilde{\varphi}^{(m, n)}(v)$. In particular, we have $\varphi^{(m, n)}\left(\overline{S_{n} x}\right)=\sum_{\eta \in S_{m}^{n}} \tilde{x} \circ \eta$, and hence $\varphi^{(m, n)} \in$ $\operatorname{Hom}_{\mathbb{Q} S_{m n}}\left(\mathbb{Q} \Omega_{m, n}, \mathbb{Q} \Omega_{n, m}\right)$ is the Schur basis element corresponding to the $\left(S_{m} \succ S_{n}\right)-\left(S_{n} \prec S_{m}\right)$-double coset $\left(S_{m} \prec S_{n}\right) \circ \sigma_{m, n} \circ\left(S_{n} \prec S_{m}\right)$ in $S_{m n}$.

In other words, if $v \in V_{m, n}$ is an $S_{n}$-minimal tuple, then $\varphi^{(m, n)}\left(\overline{S_{n} v}\right) \in \mathbb{Q} \Omega_{n, m}$ is the sum of all $\overline{S_{m} w}$, for $S_{m}$-minimal tuples in $w \in V_{n, m}$ which have the property $(\star)$ with respect to $v$. This is the original description given in [Black and List 89], where, as the main result, the following proposition is proved:

Proposition 4.1. [Black and List 89] Let $m \geq n$. If $\varphi^{(m, n)}$ is injective, then $\varphi^{(m, n-1)}$ is also injective. Thus it would be enough for proving Foulkes' conjecture to show that $\varphi^{(m, m)} \in \operatorname{End}_{\mathbb{Q} S_{m^{2}}}\left(\mathbb{Q} \Omega_{m, m}\right)$ is injective for all $m \in \mathbb{N}$.
It has already been observed in [Black and List 89], that $\varphi^{(2,2)}$ and $\varphi^{(3,3)}$ indeed are injective. Moreover, it has been shown in [Jacob 04, Section 4.2] that $\varphi^{(4,4)}$ is injective. In the rest of this paper, we will concentrate on the question of how to decide computationally whether $\varphi^{(5,5)}$ is injective or not. Due to the sheer size of this problem, it can only be tackled using particular techniques, and the answer will be given at the very end.

\section{THE COMPUTATIONAL APPROACH}

Since $\operatorname{dim}_{\mathbb{Q}}\left(\mathbb{Q} \Omega_{m, m}\right)=\left|\Omega_{m, m}\right|=\frac{\left(m^{2}\right) !}{(m !)^{m+1}}$, the matrices representing the elements of $\operatorname{End}_{\mathbb{Q} S_{m^{2}}}\left(\mathbb{Q} \Omega_{m, m}\right)$ for their natural action on $\mathbb{Q} \Omega_{m, m}$ are extremely big even for small $m$; for example, for $m=5$ we have $\left|\Omega_{m, m}\right|=$ $5,194,672,859,376 \sim 5 \cdot 10^{12}$. Hence to examine these endomorphisms, it is necessary to work in a much smaller representation of $\operatorname{End}_{\mathbb{Q} S_{m^{2}}}\left(\mathbb{Q} \Omega_{m, m}\right)$. As long as the latter is a faithful representation, the minimum polynomials of the elements of $\operatorname{End}_{\mathbb{Q} S_{m^{2}}}\left(\mathbb{Q} \Omega_{m, m}\right)$ are retained, and hence injectivity can be decided using the smaller representation. Motivated by the ideas in [Müller 03], for our computations we use the left regular representation of $\operatorname{End}_{\mathbb{Q} S_{m^{2}}}\left(\mathbb{Q} \Omega_{m, m}\right)$, which drastically reduces the size of the representing matrices: $\operatorname{dim}_{\mathbb{Q}}\left(\operatorname{End}_{\mathbb{Q} S_{m^{2}}}\left(\mathbb{Q} \Omega_{m, m}\right)\right)$ equals the character theoretic scalar product of the permutation character associated to $\Omega_{m, m}$ with itself, which can be evaluated with little effort using the computer algebra system GAP [GAP 02]. For example, for $m=5$, we find the quite moderate size $\operatorname{dim}_{\mathbb{Q}}\left(\operatorname{End}_{\mathbb{Q} S_{m^{2}}}\left(\mathbb{Q} \Omega_{m, m}\right)\right)=$ 1,856 .

According to the description of the concatenation of homomorphisms given in Section 3, we can determine the representing matrix of $\varphi^{(m, m)}$ for its left regular action, with respect to the Schur basis of $\operatorname{End}_{\mathbb{Q} S_{m^{2}}}\left(\mathbb{Q} \Omega_{m, m}\right)$, by counting. More precisely, we let $\Omega=\Omega^{\prime}=\Omega^{\prime \prime}=\Omega_{m, m}$ and $\omega_{1}=\omega_{1}^{\prime}=\omega_{1}^{\prime \prime}=\overline{S_{m} x}$, as well as $G=S_{m^{2}}$ and $H=H^{\prime}=H^{\prime \prime}=S_{m} \imath S_{m}$, and $s_{i}=t_{i}=u_{i}$ and thus $A^{(i)}=B^{(i)}=C^{(i)}$, for $1 \leq i \leq l=\operatorname{dim}_{\mathbb{Q}}\left(\operatorname{End}_{\mathbb{Q} G}(\mathbb{Q} \Omega)\right)$. Letting $s_{2}:=\sigma_{m, m} \in S_{m^{2}}$, we have $\varphi^{(m, m)}=\varphi^{(2)}$ and thus

$$
A^{(2)} \circ A^{(i)}=\sum_{k=1}^{l}\left|\omega_{1} \circ s_{i} \circ H \cap \omega_{1} \circ s_{2}^{-1} \circ H \circ s_{k}\right| \cdot A^{(k)} .
$$

Hence we have reduced the problem of studying $\varphi^{(m, m)}$ to the following tasks:

- Classify the $H$-orbits of the $G$-orbit $\Omega_{m, m}$, and thereby find corresponding representatives $\left\{s_{1}, s_{2}, \ldots, s_{l}\right\}$ of the $H$ - $H$-double cosets in $G$, where 
$s_{1}=1_{G}$ and $s_{2}=\sigma_{m, m}$; note that $\sigma_{m, m}$ is an involution.

- Determine

$$
p_{2, i, k}:=\left|\overline{S_{m} x} \circ s_{i} \circ H \cap \overline{S_{m} x} \circ s_{2}^{-1} \circ H \circ s_{k}\right|,
$$

by running through the $H$-orbit

$$
\overline{S_{m} x} \circ s_{2}^{-1} \circ H=\overline{S_{m} x} \circ \sigma_{m, m} \circ H=\bigcup_{\eta \in S_{m}^{m}} \overline{S_{m} \tilde{x}} \circ \eta,
$$

applying all representatives $s_{k}$ respectively, and classifying the resulting elements into the $H$-orbits. Note that in the computer implementation, this is done with $S_{m}$-minimal tuples representing $S_{m^{-}}$ orbits.

- Decide whether the resulting matrix $M:=$ $\left[p_{2, i, j}\right]_{i, j=1,2, \ldots, l} \in \mathbb{Z}^{l \times l}$ has full $\mathbb{Q}$-rank.

As the numerical data for the case $m=5$ given below indicate, the subtask of classifying points into $H$-orbits is still considerable. Its solution deserves a particular technique, which is a specially tailored adaptation of ideas in [Lübeck and Neunhöffer 01] and [Müller 03].

Let $U=S_{m}^{m}<S_{m} \prec S_{m}=H$ be as in Section 1. Thus, every $H$-orbit of $\Omega_{m, m}$ or $V_{m, m}$ is comprised of $U$-orbits. The basic idea now is to define $U$-minimal points in each $U$-orbit and store only those. To recognize the $H$-orbit of a point, we first find its $U$-minimalization and look that one up. To define the concept of $U$-minimality we first go back to tuples in $V_{m, m}$ :

Definition 5.1. (U-Minimal Tuple.) In $V_{m, m}$, we call the lexicographically smallest tuple in each $U$-orbit $U$ minimal. For any $v \in V_{m, m}$, we call the $U$-minimal tuple in $v \circ U$ the $U$-minimalization of $v$.

Lemma 5.2 links the concepts of $S_{m}$-minimality and $U$-minimality in $V_{m, m}$.

Lemma 5.2. If $v \in V_{m, m}$ is an $S_{m}$-minimal tuple, then its $U$-minimalization is again $S_{m}$-minimal.

Proof: By Proposition 2.2 the tuple $v$ is $S_{m}$-minimal, if and only if, for all $i, j$ with $1 \leq i<j \leq m$, the first occurrence of $i$ in $v$ is before the first occurrence of $j$ in $v$. Since the subgroup $U$ just permutes the entries within the $m$-blocks, the process of $U$-minimalization just sorts the entries in each $m$-block into ascending order.

Let $v^{\prime}$ be the $U$-minimalization of $v$ and $1 \leq i<j \leq$ $m$. If the first occurrence of $i$ and that of $j$ in $v$ are in the same $m$-block, then the same will be true after the sorting within the $m$-blocks and $S_{m}$-minimality is not violated. If they are in different $m$-blocks, the same holds, because their relative order is not changed at all.

Definition 5.3. (U-Minimal $\boldsymbol{S}_{\boldsymbol{m}}$-Orbits.) An $S_{m}$-orbit $S_{m} v \subseteq V_{m, m}$ is called $U$-minimal, if its representing $S_{m^{-}}$ minimal tuple is a $U$-minimal tuple.

Since the $S_{m}$-orbits in $V_{m, m}$ are identified with $\Omega_{m, m}$, this also defines $U$-minimal elements of $\Omega_{m, m}$. But note that this does not mean that every $U$-orbit $S_{m} v \circ U$ in $V_{m, m}$ contains exactly one $U$-minimal $S_{m}$-orbit; for example, for $m=5$, there are 2,298,891 tuples in $V_{5,5}$ which are $S_{5}$-minimal and $U$-minimal at the same time, and therefore represent $U$-minimal $S_{5}$-orbits in $\Omega_{5,5}$, while there are only $190,131 U$-orbits in $\Omega_{5,5}$ altogether. But still, the strategy sketched above works.

\section{ACTUAL COMPUTATIONS}

From here on, we concentrate on the case $m=5$, and let $G=S_{25}$ and $U=S_{5}^{5}<S_{5} 2 S_{5}=H$. It turns out that there are $623,360,743,125,120 \sim 6 \cdot 10^{14}$ tuples in $V_{5,5}$ and $5,194,672,859,376 \sim 5 \cdot 10^{12}$ points in $\Omega_{5,5}$. The $H$ orbit $\overline{S_{5} x} \circ s_{2}^{-1} \circ H$ has $(5 !)^{4}=207,360,000 \sim 2 \cdot 10^{8}$ points. The number of $H$-orbits in $\Omega_{5,5}$ is equal to $\operatorname{dim}_{\mathbb{Q}}\left(\operatorname{End}_{\mathbb{Q} G}\left(\mathbb{Q} \Omega_{5,5}\right)\right)=1,856$. Thus, it is feasible, at least by distributed computing, to run through the $H$ orbit $\overline{S_{5} x} \circ s_{2}^{-1} \circ H$ and to apply the $H$ - $H$-double coset representatives $s_{1}, s_{2}, \ldots, s_{1856}$, once we have found them. However, as already mentioned above, we have to recognize in which $H$-orbit a point $\overline{S_{5} x} \circ s_{2}^{-1} \circ h \circ s_{k}$ lies. Apart from the fact that we can not enumerate $\Omega_{5,5}$ completely, we could not even store an $H$-orbit number for each such point, as this would need at least $2 \cdot 5,194,672,859,376 \sim 10^{13}$ bytes. If we had to store every single tuple of $\Omega_{5,5}$, the situation would be even worse. To circumvent this, the notion of $U$-minimality comes into play.

In a precomputation, we classify all $2,298,891$ tuples in $V_{5,5}$ which are $S_{5}$-minimal and $U$-minimal at the same time, into the $1,856 H$-orbits in $\Omega_{5,5}$, build up a database containing these tuples and the associated $H$-orbit number, and determine suitable group elements $s_{1}, s_{2}, \ldots, s_{1856} \in G$.

A note on the classification of the $S_{5}$-minimal and $U$ minimal tuples into the $H$-orbits in $\Omega_{5,5}$ might be of interest: We first enumerate all these tuples by a standard backtrack method. Then we start putting each of 
these into a class of its own and begin applying generators of $H$ to tuples, followed by $S_{5}$-minimalization and $U$-minimalization. Whenever we observe that two tuples represent $S_{5}$-orbits in the same $H$-orbit in $\Omega_{5,5}$, we merge their classes. We repeat this until there are only 1,856 classes left. Hence, this is the distribution of $S_{5^{-}}$ minimal and $U$-minimal tuples into the $H$-orbits in $\Omega_{5,5}$. This approach turns out to work quite efficiently, and from this classification we can read off suitable elements $s_{1}, \ldots, s_{1856} \in S_{25}$.

The precomputation is implemented in the computer algebra system GAP and takes a few minutes on a modern PC. The resulting database and the elements $s_{1}, \ldots, s_{1856}$ are written out.

In the main computation, every time an $S_{5}$-orbit $S_{5} v$, represented by an $S_{5}$-minimal tuple $v$, occurs we compute the $S_{5}$-minimal tuple $v^{\prime} \in S_{5} v$ by $S_{5}$-minimalization, then we determine the $U$-minimalization $v^{\prime \prime}$ of $v^{\prime}$, which also is a $S_{5}$-minimal tuple by Lemma 5.2. The tuple $v^{\prime \prime}$ is in our database, so we can look up the $H$-orbit number of $S_{5} v^{\prime \prime}$, and, because $S_{5} v^{\prime \prime}$ is in the same $U$-orbit as $S_{5} v$, we have determined the $H$-orbit number of $S_{5} v$ by this method.

The main computation is done in a specially tailored $\mathrm{C}$ program. In this part we use distributed computing, because different instances of the program on different machines can apply different elements $s_{k}$, each having the precomputed database available. After some 14 hours of computation on about 11 modern PCs, i.e., about 150 hours of CPU time, we get the resulting matrix $M \in \mathbb{Z}^{1856 \times 1856}$, representing $\varphi^{(5,5)}$ in the left regular represention of $\operatorname{End}_{\mathbb{Q} G}\left(\mathbb{Q} \Omega_{5,5}\right)$.

The source code of the GAP and C programs that were used can be downloaded from the following web pages: http://www.math.rwth-aachen.de/ Max.Neunhoeffer/ Mathematics/foulkes.html; http://www.expmath.org/ expmath/volumes/14/14.3/Neuenhoeffer/foulkes.zip.

Finally, it remains to decide whether or not $M$ has full $\mathbb{Q}$-rank. Actually, determining the $\mathbb{Q}$-rank or even the kernel of an integer matrix of size $1,856 \times 1,856$ is not a completely trivial task. An approach to find a vector $0 \neq v \in \mathbb{Q}^{1 \times 1856}$ with $v \cdot M=0$ is to reduce $M$ modulo $p$, where $p$ is a suitable prime and find $p$-adic approximations of $v$ inductively, until a rational lift is equal to $v$. This has been described in [Dixon 82]; a sample implementation is available through the function RationalSolutionIntMat in the GAP package EDIM [Lübeck 04].

It turns out that the matrix $M$ does not have full $\mathbb{Q}$ rank. Actually, using the GAP package IntegralMeatAxe
[Müller 04], which also employs $p$-adic techniques, it is possible to compute the kernel of $M$, which turns out to have $\mathbb{Q}$-dimension 15 .

Therefore, $\varphi^{(5,5)}$ is not invertible, and hence the approach in [Black and List 89] in general does not work. Note that this does not imply a counterexample to Foulkes' conjecture. Actually Foulkes' conjecture has already been verified in [Foulkes 50] for all cases $n<m=5$. In addition, we have used the SYMMETRICA program (see [Kerber and Kohnert 92]) to verify the conjecture for all cases with $m \leq 14$ and $n \leq 4$ and for all cases with $m \leq 12$ and $n+m \leq 17$. For bigger cases, some multiplicities of simple modules in the permutation modules are greater than $2^{31}$, such that integer overflows occur on our 32 bit machines.

\section{ADDENDUM}

We have recently learned that Proposition 3.9 in [Briand 04] is a counterexample to Howe's conjecture [Howe 88], which is a strengthening of Foulkes' conjecture, and moreover is also a counterexample to Stanley's conjecture [Stanley 00, page 304], which is a generalisation of Foulkes' conjecture. We would like to thank Malek Abdesselam for pointing us in that direction.

\section{REFERENCES}

[Black and List 89] S. C. Black and R. J. List. "A Note on Plethysm." European J. Combin. 10:1 (1989), 111-112.

[Briand 04] Emmanuel Briand. "Polynômes multisymétriques." PhD diss., Université de Rennes 1 and Universidad de Cantabria. Available from World Wide Web (http://emmanuel.jean.briand.free.fr/ publications/), 2004.

[Dixon 82] John D. Dixon. "Exact Solution of Linear Equations Using p-Adic Expansions." Numer. Math. 40:1 (1982), 137-141.

[Foulkes 50] H. O. Foulkes. "Concomitants of the Quintic and Sextic up to Degree Four in the Coefficients of the Ground Form." J. London Math. Soc. 25 (1950), 205209.

[GAP 02] The GAP Group. GAP - Groups, Algorithms, and Programming, Version 4.3. Available from World Wide Web (http://www.gap-system.org), 2002.

[Howe 88] Roger Howe. " $\left(\mathrm{GL}_{n}, \mathrm{GL}_{m}\right)$-Duality and Symmetric Plethysm." Proc. Indian Acad. Sci. Math. Sci. 97: 1-3 (1988), 85-109.

[Jacob 04] Juby Jacob. "Representation Theory of Association Schemes." PhD diss., RWTH Aachen, 2004. 
[James and Kerber 81] Gordon James and Adalbert Kerber. The Representation Theory of the Symmetric Group, Encyclopedia of Mathematics and its Applications, 16. Reading, MA: Addison-Wesley Publishing Co., 1981.

[Kerber and Kohnert 92] Adalbert Kerber and Axel Kohnert. Symmetrica 1.0. Available from World Wide Web (http://www.mathe2.uni-bayreuth.de/axel/ symneu_engl.html), 1992.

[Landrock 83] Peter Landrock. Finite Group Algebras and Their Modules, London Mathematical Society Lecture Note Series, 84. Cambridge, UK: Cambridge Univ. Press, 1983.

[Lübeck 04] Frank Lübeck. GAP Package EDIM. Available from World Wide Web (http://www.math.rwth-aachen. de:8001/ Frank.Luebeck/EDIM), 2004.
[Lübeck and Neunhöffer 01] Frank Lübeck and Max Neunhöffer. "Enumerating Large Orbits and Direct Condensation." Experiment. Math. 10:2 (2001), 197-205.

[Müller 03] Jürgen Müller. "On Endomorphism Rings and Character Tables." Habilitationsschrift, RWTH Aachen, 2003.

[Müller 04] Jürgen Müller. GAP Package IntegralMeatAxe. Unpublished, 2004.

[Stanley 00] Richard P. Stanley. "Positivity Problems and Conjectures in Algebraic Combinatorics." In Mathematics: Frontiers and Perspectives, edited by V. Arnold, M. Atiyah, P. Lax, and B. Mazur, pp. 295-319. Providence, RI: Amer. Math. Soc., 2000.

Jürgen Müller, Lehrstuhl D für Mathematik, RWTH Aachen, Templergraben 64, 52062 Aachen, Germany (juergen.mueller@math.rwth-aachen.de)

Max Neunhöffer, Lehrstuhl D für Mathematik, RWTH Aachen, Templergraben 64, 52062 Aachen, Germany (max.neunhoeffer@math.rwth-aachen.de)

Received July 23, 2004; accepted February 22, 2005. 